\section{A review of Telehealth practices in Sri Lanka in the context of the COVID-19 pandemic.}

Gumindu Garuka Kulatunga ${ }^{a}$ (i), Roshan Hewapathiranab ${ }^{\text {(D) }}$, Rohana Basil Marasinghe ${ }^{c}$, Vajira H. W. Dissanayakeb (i)

\begin{abstract}
Coronavirus Disease of 2019 (COVID-19) pandemic has made healthcare staff work with physical distancing; hence Telemedicine/Telehealth is receiving renewed attention. Although Telemedicine/Telehealth is one of the major components of healthcare in most developed countries, Sri Lanka is yet to harness its full potential. The digital health ecosystem is made up of multiple interconnected solutions aimed at achieving the common goal of healthcare delivery. In the current situation, Telehealth, Telemedicine, and Internetbased Prescriptions are major components of the ecosystem. Internet-based prescriptions, which is one of the widely used Telemedicine practices, became legalised at the beginning of this century in the Western world with the incorporation of appropriate Telemedicine guidelines. Many Telemedicine solutions have emerged in Sri Lanka over the past decade including video conferencing, audio consultations, teleprescriptions and prescriptions sent by Short Message Service. These have been developed in an ad hoc manner. Therefore, the medical community, as well as the general public, must understand how Telemedicine and internet-based prescribing enable the delivery of healthcare services in the local context. In this paper, we review the current status of Telehealth services in Sri Lanka and discuss the need for regulations to ensure maximum benefit from these services.
\end{abstract}

Keywords: COVID-19, Internet-based prescriptions, Telehealth, Telemedicine, Health Informatics, Digital Health, Ecosystem, Sri Lanka

\section{TERMS OF TELEHEALTH AND TELEMEDICINE HAVE BECOME POPULAR}

Sri Lankans have been pushed into social distancing due to the COVID-19 pandemic. Healthcare services provided at a distance has become important in preventing community spread of COVID-19 through care delivery institutes, as well as to protect healthcare staff from getting infected(1). In addition, there is a need for patients with chronic noncommunicable diseases to be followed up appropriately and protected from COVID-19.

The terms, "Telehealth" and "Telemedicine" are commonly used in media and scientific literature during the management of COVID-19 throughout the world, as methods to deliver healthcare at a distance. Although the two terms are used interchangeably in the last few decades $(2,3)$ they have different connotations. Telehealth can be described as the delivery of healthcare at a distance using telecommunication technologies ${ }^{(4)}$. Common technologies that transfer information electronically include telephone, radio, fax, e-mail, the internet, video conferencing and satellite-based communications. Telehealth contains both preventive and curative aspects of healthcare delivery. Telemedicine, on the other hand, is the curative or the clinical part of Telehealth ${ }^{(3,5)}$ and can be described as 'the process of exchanging medical information from one site to another via electronic communications to improve a patient's clinical health status'(6). The types of information that can be transferred from site to site include clinical documents, laboratory results, digital photographs, electrocardiograms (ECGs), radiographs, magnetic resonance imaging (MRI) scans, computed tomography (CT) scans, real-time ultrasonography, video recordings, auscultatory sound from electronic stethoscopes and physiological examination a Ministry of Health, Nutrition \& Indigenous Medicine, Sri Lanka

b University of Colombo, Sri Lanka

c University of Sri

Jayewardenepura, Sri Lanka

Correspondence to: Dr Gumindu Garuka Kulatunga, Ministry of Health, Nutrition \& Indigenous Medicine, Sri Lanka

gumindu007@gmail.com

ORCID ID: https://orcid.org/ 0000-0003-2164-5361

Article history:

Received 08 May 2020 Revised 12 June 2020 Accepted 15 June 2020 Published 31 October 2020

\section{DOI:}

http://doi.org/10.4038/sljb mi.v11i1.8090

(C) This is an open access article licensed under a Creative Commons Attribution-Share Alike 4.0 International License (CC BYSA 4.0), which permits unrestricted use, distribution and reproduction in any medium, provided the original author and source are attributed and materials are shared under the same license.

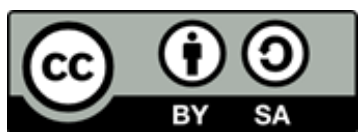


parameters such as blood pressure, heart rate, haemoglobin saturation, spirometry, etc. Figure 1 demonstrates how Telehealth, Telemedicine and internetbased prescription are interrelated in healthcare services.

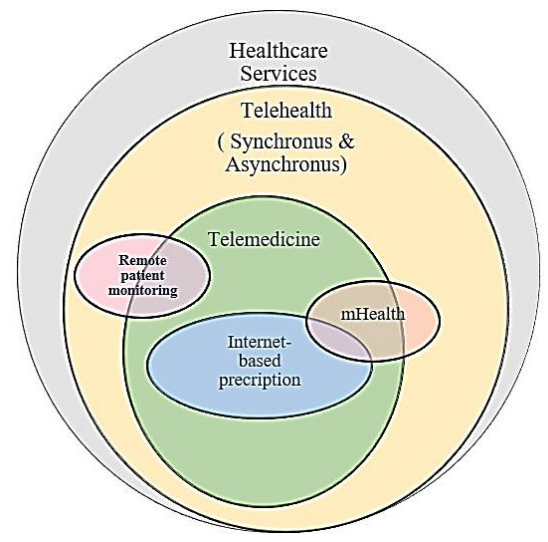

Figure 1: Relationship of internet-based prescription with Telemedicine and Telehealth

The basic concept in all Telemedicine applications is that a client of some kind (e.g., patient or health-care worker) obtains an opinion/advice from someone with more expertise in the relevant field using telecommunication, while the two parties are separated by distance, by time or by both. Clinical information transferred between these parties can be in the format of data/text (e.g., ECG, still images (e.g., X-ray or dermatology images), audio tracks (e.g., Audio call centres) or videos (e.g., ultrasound scans, Telepsychiatry) ${ }^{(7,8)}$. The information can be transferred in real-time synchronously or pre-recorded and forwarded later, as described in Table 1.

In summary, telemedicine offers remote clinical services, but the broader term of telehealth also encompasses additional nonclinical online services such as continuous medical education, professional training, and administrative meetings.

\section{DIGITAL HEALTH ECOSYSTEM}

The digital health ecosystem is where digital technology intersects with healthcare. The digital health ecosystem can be seen as a network of digital health communities ${ }^{(9)}$, which include: clients, providers, healthcare institutions and digital healthcare devices interconnected in a successfully implemented digital health environment using best practices. These include the use of information and communication technologies to empower people for monitoring and managing their health to improve the wellbeing of individuals and families.

Table 1: Common areas of information exchanged in Telemedicine practice in the world

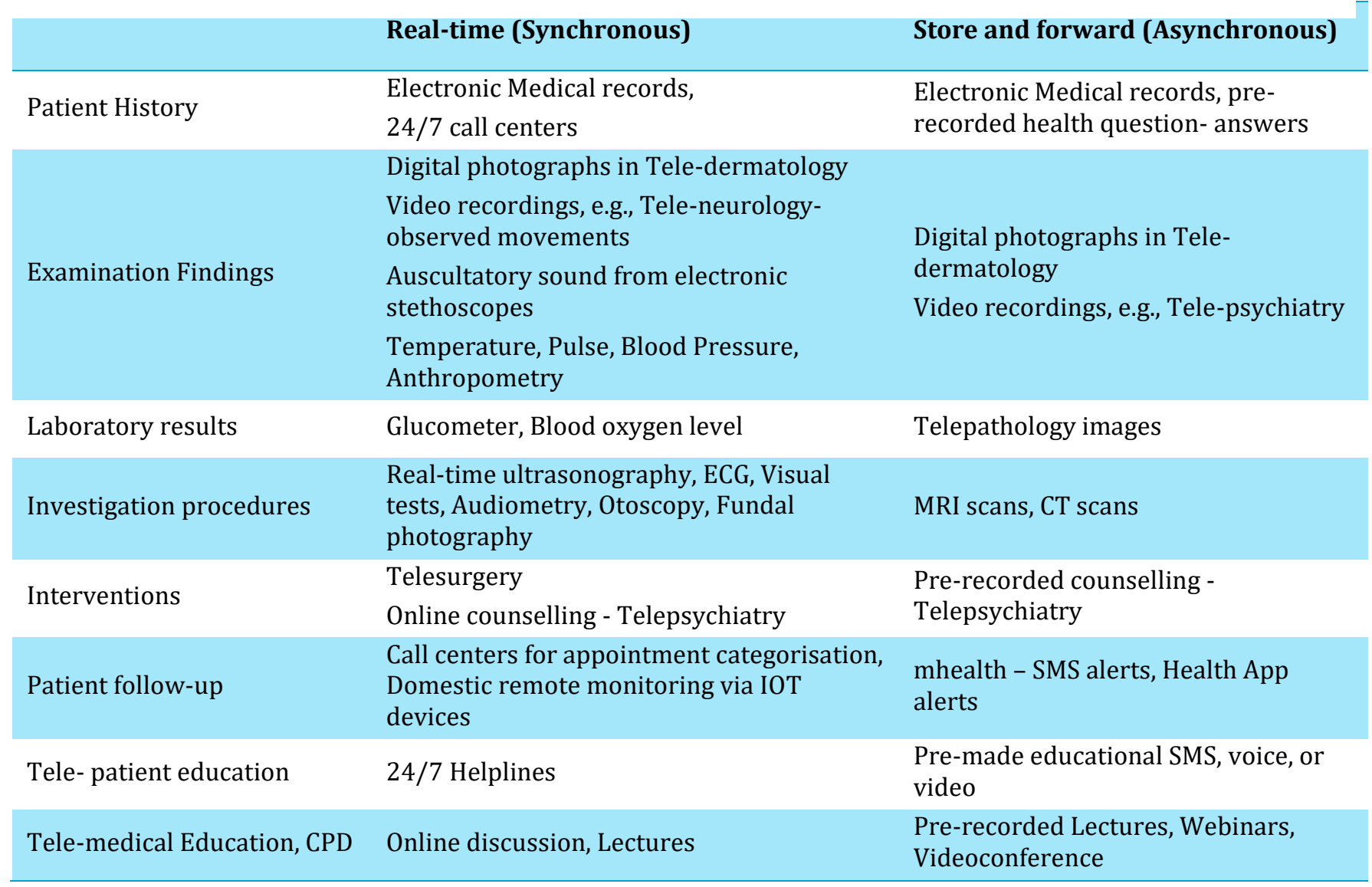


An interconnected health ecosystem will bring together stakeholders from the health sector, social care, patient groups, researchers, and the industry. This can be confined to a small geographic area or more widespread.

The main components of a digital health ecosystem include communities who trust each other and who are focused on the same goal. This includes their information or services, hardware/software technology used, participating individuals and institutions/companies, their practices, interacting platforms, and maintaining privacy, confidentiality, and security of the systems(10). Figure 2 demonstrates how Telehealth, Telemedicine and internetbased prescription have become components of this digital health ecosystem.

\section{WHY DO WE NEED TELEMEDICINE?}

There are two instances when Telemedicine becomes important. The first instance is when there is no alternative other than Telemedicine in the management of medical emergencies in remote environments such as rural communities, ships, or aeroplanes. The second instance is when Telemedicine is better than existing conventional services in the delivery of healthcare.

Although it seems that Telemedicine can only be relevant for nations with vast geographical expanses, even in a citysize country like Singapore, Telemedicine has a unique role to play ${ }^{(9)}$. Even in countries with abundant healthcare resources, including a high amount of clinicians, technology-assisted health delivery such as Telemedicine can play a major role in reaching underserved populations ${ }^{(10)}$.

\section{WHY ARE TELEHEALTH SERVICES IMPORTANT IN THE CURRENT COVID-19 PANDEMIC?}

In various parts of the world, public urgency and uncontained spread of COVID-19 have caused overcrowding of respiratory units in hospitals with both symptomatic and anxious asymptomatic patients. This could strain healthcare staff as well as affect medical supply chains(11). Previous studies have described the

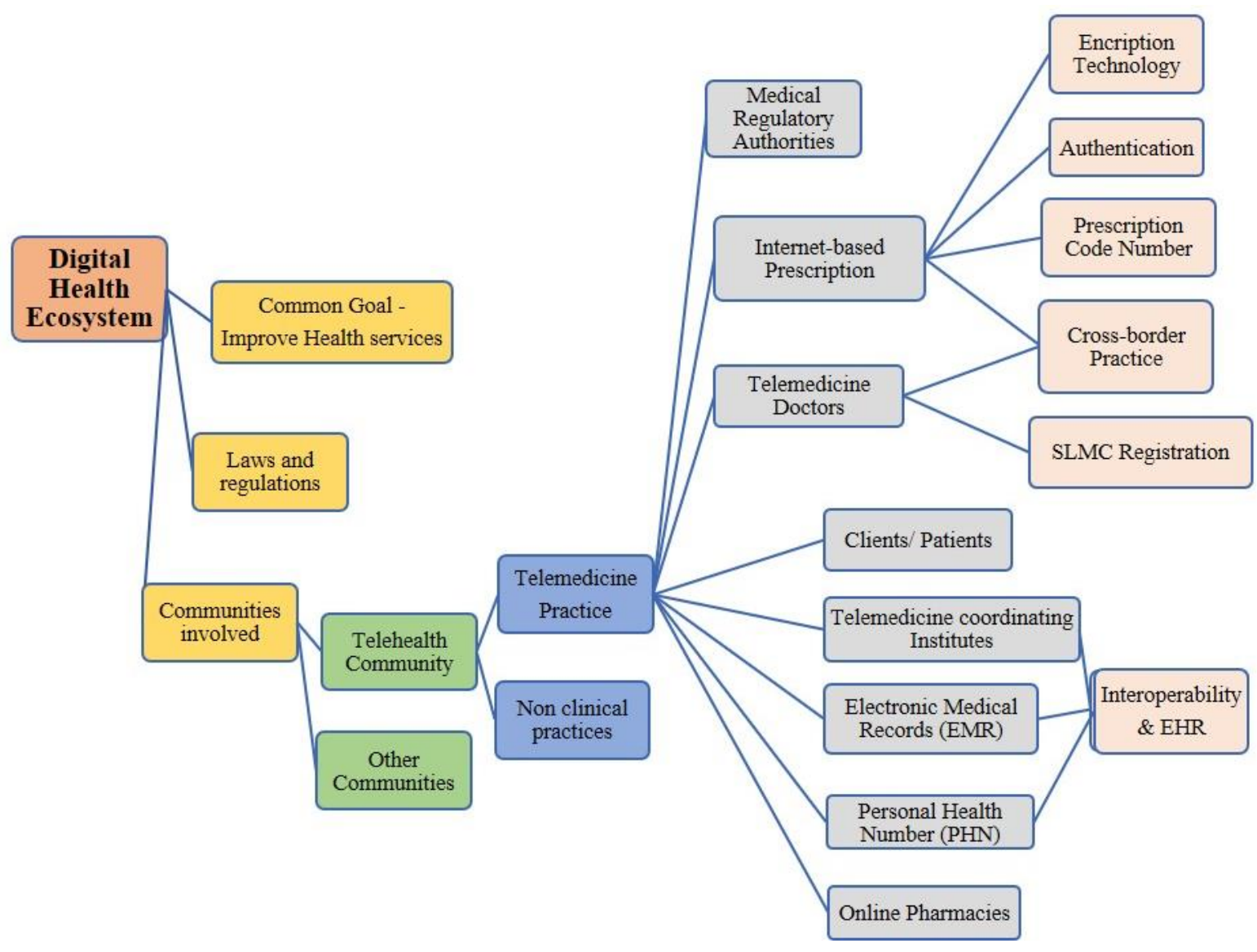

Figure 2: The place of Telemedicine and Internet-based prescription within the digital health ecosystem 
potential benefits of using Telehealth in similar situations ${ }^{(12) .}$

Telehealth services can help COVID-19 management by screening high-risk groups and identifying potential cases, assisting in the provision of inward patient care, and remotely following up people on self-quarantine and patients discharged from hospitals.

Telehealth systems can be used to prevent overcrowding of healthcare institutions. Besides, the use of Telehealth would reduce human to human exposures and protect healthcare workers and patients from a wide range of infectious diseases and ensure that essential human and other medical resources are reserved for patients who need them most. Further, Telehealth can be used for the follow-up of patients with chronic disorders such as diabetes or heart diseases who are at high risk for COVID-19 related complications by reducing physical clinic visits.

\section{EVOLUTION OF TELEHEALTH AND TELEMEDICINE}

The scientifically presented first case of using communication technology for clinical care was reported in The Lancet in 1897, where a telephone conversation was used to diagnose a child with croup ${ }^{(13)}$. Since then, there had been many milestones in the evolution of Telehealth up to now ${ }^{(14)}$.

The prefix "Tele" was first used in the medical context by Einthoven, a Dutch physician in 1905 when he reported about the telephonic transmission of electrocardiographic images and called it a "Tele-cardiogram"(11). In 1910, the telephone was used to transmit amplified sounds from a stethoscope using telecommunication networks. After the invention of radio waves, Telehealth services acquired rapid growth. In 1920, the Seaman's Church Institute of New York became one of the first organisations to provide medical care using radiofrequency. By 1935, International Radio Medical Centre, headquarters was set up in Rome, Italy.

A major development of the clinical aspect of Telehealth, which is Telemedicine, came with the introduction of closed-circuit television by late 1950s. Further, the introduction of television by the late1950s permitted interactive consultations. As early as 1964, a two-way closed-circuit television system was set up between psychiatric institutes and mental hospitals within the USA.
The system permitted interactive teleconsultations between general practitioners and hospital specialists. The term "Telemedicine" was introduced to the world in the 1960s(12). The term "Telemedicine" was introduced by Bird and his colleagues in 1969 to describe the delivery of medical care without the usual patient-physician confrontation $^{(15)}$. Later, Bannet and associates introduced the word "Telehealth" in 1978 to broaden the scope of Telemedicine with the incorporation of patient/clinician

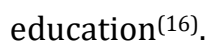

With the introduction of mobile phones and satellite communication, Telehealth stepped up into the stage of mobile Telehealth. The expansion of the internet facilitated an unchallengeable boost to Telehealth. Telecommunication technologies, including the internet, now facilitates the delivery of innovative healthcare services and products. Telesurgery is another breakthrough in Telemedicine. In 2001, surgeons in New York, USA performed surgery on a woman in France using high definition communication equipment and a highspeed internet connection, which was called 'Lindbergh Operation'. Since the beginning of the 21st century, many doctors, other healthcare providers and technical experts throughout the world dedicated their time to deliver Telehealth services(17), and Telehealth became one of the fastest-growing sectors of medical services. Telemedicine is a continuously developing field and has been considered as a subtopic of medical informatics(17) for the last few decades.

\section{WHAT IS AN INTERNET-BASED PRESCRIPTION?}

Internet-based prescription is a patient receiving a soft copy version of a prescription through the internet instead of a handwritten or a printed prescription. Throughout the world, prescriptions are being sent online frequently. There is a distinction to be made between Internet dispensing and Internet prescribing(18,19). 'Internet drug dispensing/ e-prescribing' means delivery of a valid webbased prescription electronically via the internet to the pharmacy from the point of care, after a regular face to face consultation with the doctor guiding the patient to collect drugs directly without a paper-based prescription. 'Internet-based drug prescribing or remote prescribing', on the other hand, is the act of online prescribing, which would entitle the patient to receive the drugs from the pharmacy, following a distant teleconsultation (without a 
person-person contact between a health provider and patient face to face). The position of internet-based prescription in the ecosystem is shown in Figure 2.

\section{TRANSFORMATION OF THE CONVENTIONAL PRESCRIPTION INTO AN INTERNET-BASED PRESCRIPTION}

Prescription for medications is frequently associated with medical consultations. The practice of prescribing medicine among humans traces back in history to the times of the Sumerians in around 2,000 BC. Further, there are records of prescribing and dispensing medication during the Han Dynasty in China, in ancient Greece, as well as the Islamic Golden Age in Iraq ${ }^{(20)}$. Documented accounts on medical prescription can be found in the Egyptian Papyri. Egyptian Papyrus 'Ebers' from 1550 B.C containing 811 complete medical prescriptions for various ailments is the largest of such and the best-preserved. It was discovered at Thebes, in 1862, by Georg Ebers(21,22). The symbol of' $\mathrm{Rx}^{\prime}$, which represents a prescription also originated from ancient Egypt where it had been written on ancient prescriptions, symbolising a prayer for the god of healing. Further, the symbol of ' $\mathrm{Rx}$ ' was an abbreviation for the Latin word 'recipe'(22). In ancient days, this 'recipe' document contained directions for the pharmacists on making up and preparing medications.

In the current era, a drug prescription can be issued only by a registered or licensed medical practitioner. It is a recognised fact that medical professional standards are of paramount importance as it can lead to serious consequences otherwise. However, in the ancient world, the practice of medicine was open to all, without any registration. The 'Hippocratic oath' was a private contract between a master and a pupil and not a legal document of registration. At that time, there were no regulations to define the role of the physician. The need for a registration emerged in ancient Iraq in 931 A. D. when the Baghdad ruler decided that no one should practice medicine in the capital unless he has been authorised by a specified senior physician(23). First regulations in Europe were issued in connection with the school of Salerno, Italy, the first medical school in the Western world in the 10th century. A more detailed set of documented regulations concerning physicians were made during the reign of Hohenstaufen Emperor Frederick II between 1231 to 1240 in ancient Europe(23). These licensing regulations drew up strict standards for medical practice and pharmacies.

During the era of the post-industrial revolution, the healthcare industry, unlike other industries, trailed behind in embracing innovations in information technology since the medical practice was bound by many regulations and ethics. Back in the 1960s, pharmacies were at the forefront of automation in healthcare. However, e-prescribing only became prominent with the passage of the Medicare Prescription Improvement, and Modernization Act of 2003 in the USA ${ }^{(24)}$. Thereafter, electronic prescribing became legal in all 50 states of the USA in $2007^{(25)}$. Since then, eprescribing technology has taken hold as an advancement over handwritten drug treatment orders. United States Medical regulation passed in 2008 and 2009, permits insurance companies in the US to give incentives to physicians for using electronic prescribing technology(24). The evolution of prescription is graphically demonstrated in Figure 3.

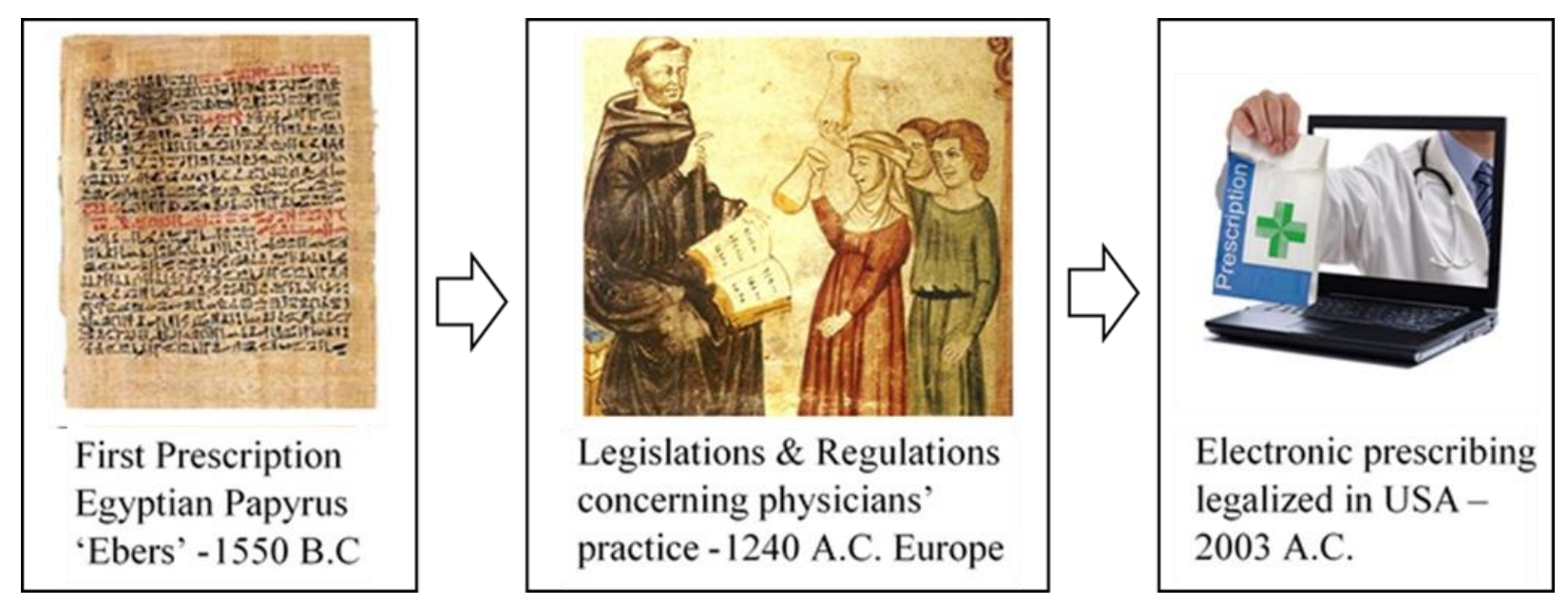

Figure 3: Evolution in the method of prescribing across centuries

(Image Credit: Papyrus image: National Medical Library, National Institutes of Health, USA: Medieval-physician image: Swilliams (talk / contribs): Laptop image: (CAlarmy Stock photo) 
PROVEN ADVANTAGES OF TELEHEALTH, TELEMEDICINE, AND INTERNET-BASED PRESCRIPTIONS

As Telehealth continues to develop rapidly, policymakers in healthcare are becoming increasingly interested in its cost/benefit analysis. The major attractiveness of Telemedicine is that it removes the time-consuming factor of 'transport' that is needed for a face-to-face consultation between the patient and the health professional at a healthcare institute. This is important in the time of COVID-19 pandemic where movement is risky as well as restricted.

Telemedicine will help to bridge the gap that exists between the patients and the doctor. Not only will the patient feel more in control of his/her health, but also the doctor who is treating will get more time to review the individual case, possibly supported by external expertise if needed. Further, in rural areas, it improves access to health services minimising travel time for patients and health workers. In urban areas, it improves access to health information as well as services, which will save time. Regardless of the few exceptions on restricted and controlled drugs, internet prescribing poses no real health risk to consumers ${ }^{(18)}$ and provide a wide range of proven advantages ${ }^{(26)}$.

Telehealth research in the past had shown that patient satisfaction is greater than $80 \%$, and the reason for satisfaction possibly due to quick appointment dates or less time spent on travelling(27). Telemedicine has demonstrated its effectiveness in improving the management of chronic conditions, improving decision making in acute situations, compensating for scarcity of specific local healthcare services, providing care from rare specialities(28), reducing health costs and preventing hospitalisations and emergency room use(29). Telehealth can create a virtual community facilitating collaboration between professionals and patients, leading to the development of a care plan for individual patients(28). Research has confirmed that there is a $45 \%$ reduction in mortality rate and a $20 \%$ reduction in admissions to the emergency department by using Telehealth(30).

Several Randomised Control Trials (RCT) have shown the effectiveness of Telemedicine applications over conventional methods. Table 2 shows some of the positive results found in the RCT report in 2019. However, only one RCT study has been performed in Sri Lanka during the last decade. In the above trial, Telehealth intervened group achieved significant improvements in reducing suicidal ideation and depression(31).

\section{EMERGING RISKS OF TELEMEDICINE AND INTERNET-BASED PRESCRIPTION}

As in the case of other new technological innovation, many potential risks will emerge with extensive spread use of Telemedicine. There can be issues on, reduced or nonacceptable standard of care, poor control of prescribing controlled medications, reduce or nongenuine doctor-patient relationship, deficient service reimbursement policies, risks in cybersecurity/privacy protection, risks of getting prescriptions by a physician living outside patient's country or without the license(26). Further, there is a risk of "doctor shopping" where customers seeking prescription drugs for illegal use, will target doctors who prescribe without proper screening(32). These have to be prevented by appropriate legislation.

Therefore, many countries have modified their medical acts to keep up with the changing technologies, including Telehealth. Some countries have developed standards and policies on Telehealth, and a few, such as USA, Australia, France, Germany, and Singapore, have gone a step further and have included Telemedicine regulations into their national legislation.

\section{THE CURRENT STATUS OF TELEHEALTH SERVICES IN SRI LANKA}

Telehealth has been around for a decade in the country(33), but its adoption among the general public has been relatively low. The COVID-19 Pandemic is quickly changing the status quo, and Telehealth services are expanding rapidly. Even the Ministry of Health has developed mobile applications to expand Telehealth services in Sri Lanka(34). However, there were many governmental as well as non-governmental organisations that provide Telehealth services(35) even before the pandemic. However, scientific publications related to Telehealth in Sri Lanka are minimum. A Telehealth study done in Sri Lanka a few years ago reported that most patient requests for interventions were regarding behavioural and psychiatric disorders and genitourinary disorders(33). An RCT done on Sri Lanka in suicide prevention has shown a superior effect of the intervention over the conventional method(31). The success of telepsychiatry and other related disciplines may account for the ability of Telehealth to elicit sensitive and personal 
Table 2: Randomised Control Trials reported on Telemedicine interventions in 2019

\section{Topic}

Telemedicine for Continuous Positive

Airway Pressure in Sleep Apnoea: A

Randomised, Controlled Study (36)

\begin{abstract}
Telepsychiatry- Based Care for the
Treatment Follow-Up of Iranian War

Veterans with Post-Traumatic Stress

Disorder: A Randomized Controlled

Trial (37).
\end{abstract}

Area

Continuous positive airway

pressure (CPAP) management in

obstructive sleep apnoea syndrome

patients

Results of the group participated in Telemedicine intervention

Improvement in sleep-related

quality of life

Management of Post-Traumatic

Stress Disorder

Increased patient satisfaction, completion of therapy sessions, and reduction in treatment costs
Randomised clinical trial of accelerated enhanced recovery after minimally invasive colorectal cancer surgery (RecoverMI trial) (38)

Effect of telerehabilitation on longterm adherence to yoga as an antihypertensive lifestyle intervention: Results of a randomised controlled trial (39)

Psychological therapies (remotely delivered) for the management of chronic and recurrent pain in children and adolescents $(40)$

A randomised trial of Telemedicine for migraine management ${ }^{(41)}$
Post-operation recovery after minimally invasive colorectal cancer surgery
Reduced duration of hospital stay, preserving patients' quality of life and satisfaction
Adherence to yoga as an antihypertensive intervention
Improves adherence to yoga, maintaining achieved health benefits in the long run

\section{Efficacy of psychological therapies} delivered remotely for the management of chronic pain in children and adolescents
Headache severity was reduced post-treatment

Convenience was rated high and visit times were shorter in the Telemedicine group
Effect of lifestyle-focused text messaging on risk factor modification in patients with diabetes and coronary heart disease: A sub-analysis of the TEXT ME study(42)
Migraine management
Risk factor modification in patients with coronary heart disease and Type 2 diabetes mellitus
Lifestyle-focused text messaging resulted in significant risk factor reduction
Service delivery to participants of public health smoking cessation clinic
Increases the abstinence rate and has favourable effects on follow-up. effects on abstinence rates in a randomised controlled study ${ }^{43)}$.

Weight management Telehealth intervention for overweight and obese rural cardiac rehabilitation participants: A randomised trial (44)
Weight management intervention for a group of rural cardiac rehabilitation patients
Improved weight management outcomes 
information that patients may not like to discuss face-toface with their doctor in an out-patient department setting. However, in the Western world, reasons for online preference can be due to above mentioned social factors $^{(45)}$ and due to frustration or disappointment regarding the previous face to face consultations, convenience and ability to maintain patient anonymity(46,47).

Several web-based online pharmacies have been established in Sri Lanka during the past few years to cater to electronic prescriptions as well as written prescriptions. The lack of clarity in drug regulation makes it possible for these services to dispense drugs using photographed/scanned and uploaded prescriptions ${ }^{(48,49)}$. In response to the COVID-19 lockdown situation in Sri Lanka, even the government had to resort to similar practices to ensure the delivery of drugs to patients(50).

\section{SRI LANKAN GUIDELINES AND LEGISLATIONS RELATED TO TELEHEALTH}

Even though many Telehealth practices are emerging in Sri Lanka, there has been no attempt to streamline and upgrade the process. Sri Lanka does not have a national agency committed to develop and promote Telehealth/Telemedicine. Also, there are no policies or regulations on the practice of Telehealth/Telemedicine ${ }^{(51,52)}$.

The Ministry of Healthcare and Indigenous Medical Services is responsible for healthcare policymaking, planning and regulation of health-related matters in Sri Lanka. The Sri Lanka Medical Council (SLMC), established in 1998 under the Medical (Amendment) Act No. 40 of 1998 which replaced the former Ceylon Medical Council that was formed in 1924, is the legal body responsible for regulating individual doctors, including licensing, developing codes of conduct and dealing with complaints against doctors. According to the Medical Ordinance, which was enacted in 1924, a doctor must obtain a medical license (specified by a license number) to practice medicine in any part of Sri Lanka. Section 29 of Medical Ordinance no 26 of 1927 amended in 1988(53) stipulate that a medical practitioner should be registered in Sri Lanka Medical Council (SLMC) to provide patient treatment, including prescriptions. However, the act does not mention anything about the possibility or right to offer medical service remotely.

Regulation of Telehealth services is essential because the
Sri Lankan public will be exposed to non-Sri Lankan doctors providing cross border medical services in the future.

National Medicines Regulatory Authority of Sri Lanka is the regulator that registers medications to be used in Sri Lanka. It also regulates drug distribution and prescribing methodologies in Sri Lanka. The National Medicines Regulatory Authority Act, No. 5 Of 2015(54) make up the related legislation used to regulate distribution and sale of drugs/medications in Sri Lanka. However, these laws do not have provisions to regulate internet-based prescriptions. The act refers only to written prescriptions issued by registered doctors. The prevailing legal act which is used for medical as well as nonmedical document interchange electronically within Sri Lanka is the Electronic Transactions Act - No. 19 of 2006(55). This act authorises the use of electronic filing of documents and assists domestic and international electronic commerce. Even the latest amendment to the Act, Electronic Transactions (Amendment) Act, No. 25 of 2017(56), is silent on internet-based prescriptions.

Even though there are many guidelines and protocols for the practice of different medical specialities in Sri Lanka, there are no guidelines or protocols on Telehealth. Ministry of Health's National eHealth Guidelines(57) and the National Policy on Health Information(58) does not provide any guideline for Telehealth as well as internet prescriptions in Sri Lanka. There had been few scientific publications describing Telehealth services in Sri Lanka(52,59-61). There is, however, a lack of scientific research in the field of regulation of Telehealth services in the country.

Same as Sri Lanka, the South East Asian region too is lagging behind the rest of the world in developing policies on the expansion of Telemedicine. Around 40\% of countries in Europe have a national Telemedicine policy, whereas only $10 \%$ to $15 \%$ of countries in South-East Asia have such policy(62). Telehealth practitioners have been following the usual codes of ethics for the medical profession in combination with existing general regulations when there is no direct Telehealth related legislation(63). However, many developed countries have identified the need to streamline Telehealth services, more than a decade ago(17). The Federation of State Medical Boards (FSMB) of the United States of America announced that Telemedicine as the most important medical regulatory topic that needs to be addressed in $2016^{(64)}$. 
HOW TO ADDRESS THE FUTURE NEEDS OF THE SRI LANKAN TELEHEALTH SECTOR WITH A FOCUS ON INTERNET-BASED PRESCRIPTIONS

Telehealth and internet-based prescription practice are expanding in Sri Lanka. However, there is a deficiency in the availability of guidelines for the provision of Telehealth services. Therefore, it is a current national need to evaluate citizen's Telehealth needs and current
Telehealth practices in the country, including Internetbased prescriptions. There is a need to develop a national framework for Telehealth. It is essential to update guidelines and health-related acts to cover the registration criteria of doctors who are prescribing over the internet. Prescribing protocols for controlled medications should also be established. These will promote the provision of Telehealth services in a controlled manner for the benefit of patients in the country.

\section{REFERENCES}

1. Moazzami B, Razavi-Khorasani N, Dooghaie Moghadam A, Farokhi E, Rezaei N. COVID-19 and telemedicine: Immediate action required for maintaining healthcare providers well-being. Journal of Clinical Virology: The Official Publication of the Pan American Society for Clinical Virology. 2020;126:104345.

2. Bashshur R, Shannon G, Krupinski E, Grigsby J. The taxonomy of telemedicine. Telemedicine Journal and E-Health: The Official Journal of the American Telemedicine Association. 2011 08;17(6):484-494.

3. Van Dyk L. A Review of Telehealth Service Implementation Frameworks. International Journal of Environmental Research and Public Health. 2014 01-23;11(2):1279-1298.

4. White LAE, Krousel-Wood MA, Mather F. Technology Meets Healthcare: Distance Learning and Telehealth. The Ochsner Journal. 2001 01;3(1):22-29.

5. Sood S, Mbarika V, Jugoo S, Dookhy R, Doarn CR, Prakash N, et al. What is telemedicine? A collection of 104 peer-reviewed perspectives and theoretical underpinnings. Telemedicine Journal and E-Health: The Official Journal of the American Telemedicine Association. 2007 10;13(5):573-590.

6. Voran D. Telemedicine and Beyond. Missouri Medicine. 2015;112(2):129-135.

7. Roine R, Ohinmaa A, Hailey D. Assessing telemedicine: a systematic review of the literature. Canadian Medical Association Journal. 2001;165(6):765-771.

8. Tulu B, Chatterjee S, Laxminarayan S. A Taxonomy of Telemedicine Efforts with Respect to Applications, Infrastructure, Delivery Tools, Type of Setting and Purpose. In: Proceedings of the Proceedings of the 38th Annual Hawaii International Conference on System Sciences (HICSS'05) - Track 6 - Volume 06 [Internet]. USA: IEEE Computer Society; 2005. p.

Rockwell KL, Gilroy AS. Incorporating telemedicine as part of COVID-19 outbreak response systems. The American Journal of Managed Care. 2020;26(4):147-148.

12. Hollander JE, Carr BG. Virtually Perfect? Telemedicine for Covid-19. New England Journal of Medicine. 2020 04-30;382(18):1679-1681.

13. Ministry of Health, Singapore. National Telemedicine Guidelines (NTG) for Singapore [Internet]. https://www.moh.gov.sg. 2015. Available from: https://www.moh.gov.sg/content/dam/moh_web/ Publications/Guidelines/MOH\%20Cir\%2006_2015 _30Jan15_Telemedicine\%20Guidelines\%20rev.pdf

14. Young JD, Badowski ME. Telehealth: Increasing Access to High Quality Care by Expanding the Role of Technology in Correctional Medicine. Journal of Clinical Medicine. 2017 02-13;6(2):20.

15. Darkins AW, Cary MA. Telemedicine and Telehealth: Principles, Policies, Performances and Pitfalls. Springer Publishing Company; 2000.

16. Craig J, Patterson V. Introduction to the practice of telemedicine. Journal of Telemedicine and Telecare. 2005;11(1):3-9.

17. Einthoven W. Het telecardiogram [The telecardiogram]. Nederlands Tijdischrift voor Geeneskunde. 1906;(53):601-615. 
18. Angaran DM. Telemedicine and telepharmacy: current status and future implications. American journal of health-system pharmacy: AJHP: official journal of the American Society of Health-System Pharmacists. 1999 07-15;56(14):1405-1426.

19. Bird K. Concept and practice. In: Telemedicine, explorations in the use of telecommunications in health care. Springfield: Charles Thomas; 1975. p. 89-125.

20. Bennet A, Rappaport W, Skinner E. Telehealth handbook. Washington DC: PHS publication, U.S. Department of Health, Education, and Welfare; 1978.

21. Iserson KV. Telemedicine: a proposal for an ethical code. Cambridge Quarterly of Healthcare Ethics. 2000;9(3):404-406.

22. Friedman KM. Internet prescribing limitations and alternatives. Annals of Health Law. 2001;10:139178.

23. Terry NP. Prescriptions sans frontières (or how I stopped worrying about Viagra on the Web but grew concerned about the future of healthcare delivery). Yale Journal of Health Policy, Law, and Ethics. 2004;4(2):183-272.

24. Venosa A. History of The Pharmacy: How Prescription Drugs Began And Tranformed Into What We Know Today [Internet]. medicaldaily. 2016. Available from: https://www.medicaldaily. com/pharmacy-prescription-drugs-378078

25. Clendening L. Source book of medical history [Internet]. New York: Dover Publications; 1960. Available from: https://books.google.lk/books? $\mathrm{hl}=$ en \&lr=\&id=Ne0hDgAAQBAJ\&oi=fnd \&pg=PA1\&d $\mathrm{q}=$ history+of +medical+prescriptions\&ots=6JvU4P8 21I\&sig=iDgC8MNa5mxZWJaG8qU7SXq83N0\&redi r_esc=y\#v=onepage $\& q=$ history $\% 20$ of $\% 20$ medical $\% 20$ prescriptions $\& \mathrm{f}=$ false

26. Atalar A. The history of prescriptions [Internet]. The Pharmaceutical journal. 2012. Available from: https://www.pharmaceutical-journal.com/opinion /blogs/the-history-of-prescriptions/11102501.blog ?firstPass=false

27. Sigerist HE. The history of medical licensure. Journal of the American Medical Association. 1935;104(13):1057-1060.

28. Greene J, Yellowlees PM. Electronic and Remote Prescribing: Administrative, Regulatory, Technical, and Clinical Standards and Guidelines, April 2013.
Telemedicine and e-Health. 2013 11-27;20(1):6374.

29. 10 Years of Interoperability in Action: A Historical Look at E-Prescribing [Internet]. Prescription Accuracy - Intelligence in Action \textbar Surescripts. 2015. Available from: https:// surescripts.com/news-center/intelligence-in-action /prescription-accuracy/10-years-of-interoperabilit y-in-action-a-historical-look-at-e-prescribing

30. Langbecker D, Caffery LJ, Gillespie N, Smith AC. Using survey methods in telehealth research: A practical guide. Journal of Telemedicine and Telecare. 2017 10;23(9):770-779.

31. Charrier N, Zarca K, Durand-Zaleski I, Calinaud C. Efficacy and cost effectiveness of telemedicine for improving access to care in the Paris region: study protocols for eight trials. BMC Health Services Research. 2016 02-08;16:45.

32. Kaldy J. Telehealth enables senior care pharmacists to reach beyond facility walls. The Consultant Pharmacist: The Journal of the American Society of Consultant Pharmacists. 2005 07;20(7):558-560, 563-7.

33. Caceres C, Gomez EJ, Garcia F, Chausa P, Guzman J, Del Pozo F, et al. A Home Integral Telecare System for HIV/AIDS Patients. Studies in Health Technology and Informatics. 2005;114:23-29.

34. Steventon A, Bardsley M, Billings J, Dixon J, Doll H, Hirani S, et al. Effect of telehealth on use of secondary care and mortality: findings from the Whole System Demonstrator cluster randomised trial. BMJ (Clinical research ed). 2012 06-21;344:e3874.

35. Marasinghe RB, Edirippulige S, Kavanagh D, Smith A, Jiffry MTM. Effect of mobile phone-based psychotherapy in suicide prevention: a randomized controlled trial in Sri Lanka. Journal of Telemedicine and Telecare. 2012 04;18(3):151-155.

36. Schoch OD, Baty F, Boesch M, Benz G, Niedermann J, Brutsche MH. Telemedicine for Continuous Positive Airway Pressure in Sleep Apnea: A Randomized, Controlled Study. Annals ATS. 2019 Jul 16;AnnalsATS.201901-0130C.

37. Haghnia Y, Samad-Soltani T, Yousefi M, Sadr H, Rezaei-Hachesu P. Telepsychiatry- Based Care for the Treatment Follow-Up of Iranian War Veterans with Post-Traumatic Stress Disorder: A Randomized Controlled Trial. Iranian Journal of Medical Sciences [Internet]. 2019 Jul [cited 2019 Sep 3];44(4). 
Available from: http://doi.org/10.30476/ijms .2019 .44944

38. Bednarski BK, Nickerson TP, You YN, Messick CA, Speer B, Gottumukkala V, et al. Randomized clinical trial of accelerated enhanced recovery after minimally invasive colorectal cancer surgery (RecoverMI trial). Br J Surg. 2019 Sep;106(10):1311-8.

39. Sarah S, Wolfgang M-B, Claudia P. Effect of telerehabilitation on long-term adherence to yoga as an antihypertensive lifestyle intervention: Results of a randomized controlled trial. Complementary Therapies in Clinical Practice. 2019 May;35:148-53.

40. Fisher E, Law E, Dudeney J, Eccleston C, Palermo TM. Psychological therapies (remotely delivered) for the management of chronic and recurrent pain in children and adolescents. Cochrane Pain, Palliative and Supportive Care Group, editor. Cochrane Database of Systematic Reviews [Internet]. 2019 Apr 2 [cited 2019 Sep 3]; Available from: http://doi.wiley.com/10.1002/14651858.CD01111 8.pub3

41. Friedman D, Rajan B, Seidmann A. A randomized trial of telemedicine for migraine management. Cephalalgia [Internet]. 2019 Aug 26 [cited 2019 Sep 3]; Available from: https://www.ncbi.nlm.nih.gov /pubmed/31450969

42. Haider R, Hyun K, Cheung NW, Redfern J, Thiagalingam A, Chow CK. Effect of lifestyle focused text messaging on risk factor modification in patients with diabetes and coronary heart disease: A sub-analysis of the TEXT ME study. Diabetes Res Clin Pract. 2019 Jul;153:184-90.

43. Durmaz S, Ergin I, Durusoy R, Hassoy H, Caliskan A, Okyay P. WhatsApp embedded in routine service delivery for smoking cessation: effects on abstinence rates in a randomized controlled study. BMC Public Health. 2019 Dec;19(1):387.

44. Barnason S, Zimmerman L, Schulz P, Pullen C, Schuelke S. Weight management telehealth intervention for overweight and obese rural cardiac rehabilitation participants: A randomised trial. J Clin Nurs. 2019 May;28(9-10):1808-18.

45. Manchikanti L. National drug control policy and prescription drug abuse: facts and fallacies. Pain Physician. 2007 05;10(3):399-424.

46. Kulatunga GG, Hewageegana NR, Perera MK, Amunugama S. Understanding the Tele-health needs of Suwásariya (Health Net) users. Indian Journal of Medical Informatics. 2014;8(2):96-99.
47. 'MyHealth Sri Lanka' mobile app launched to consult doctors [Internet]. Dailymirror online news. 2020. Available from: http://www.dailymirror.lk/ breaking_news/'MyHealth-Sri-Lanka'-mobile-applaunched-to-consult-doctors/108-186421

48. Razick S. How Sri Lanka's Healthcare Industry Is Experimenting With Technological Solutions [Internet]. roar.media. 2019. Available from: https://roar.media/english/life/in-the-know/howsri-lankas-healthcare-industry-is-experimentingwith-technological-solutions/

49. Economics E. Regulatory approaches to telemedicine. 2018 07-21; Available from: https://www.gmc-uk.org/about/what-we-do-andwhy/data-and-research/research-and-insightarchive/regulatory-approaches-to-telemedicine

50. Umefjord G, Hamberg K, Malker H, Petersson G. The use of an Internet-based Ask the Doctor Service involving family physicians: evaluation by a web survey. Family practice. 2006;23(2):159-166.

51. Modahl M, Meinke S. Telehealth Index: 2015 Consumer Survey [Internet]. United States; 201501. Available from: https://www.americanwell.com /infographic-telehealth-index-2015-consumersurvey/

52. Online pharmacy in Sri Lanka \textbar HealthNetBuy [Internet]. Available from: https:// www.healthnetbuy.com/

53. Wijekoon CB. Sri Lanka's first ever online-pharmacy unveiled. Daily News [Internet]. 2016 04-01; Available from: http://www.dailynews.lk/2016/04 /01/business/78015

54. Arrangements made to home-deliver medications [Internet]. Sri Lanka News: ColomboPage - Original News from Sri Lanka. 2020. Available from: http://www.colombopage.com/about_us.php

55. World Health Organization. ehealth country profiles 2009 -Sri Lanka [Internet]. 2009. (Global Observatory for eHealth (GOe)). Available from: https://www.who.int/goe/publications/atlas/2009 /lka.pdf?ua=1

56. Rampatige R, Abusayeed MH, Galappaththi H. Global Observatory for eHealth (GOe) Survey in Sri Lanka. Sri Lanka Journal of Bio-Medical Informatics. 2010;1(1).

57. Medical Ordinance [Internet]. Parliament of the Democratic Socialist Republic of Sri Lanka; 1927. Available from: https://www.lawnet.gov.lk/1948 
/12/31/medical-practitioners-pharmacistsmidwives-and-nurses-3/

58. National Medicines Regulatory Authority Act. Vol. 5. Parliament of the Democratic Socialist Republic of Sri Lanka; 2015.

59. Electronic Transactions Act. Parliament of the Democratic Socialist Republic of Sri Lanka; 2006.

60. Electronic Transactions (Amendment) Act [Internet]. Parliament of the Democratic Socialist Republic of Sri Lanka; 2017. Available from: https://srilankalaw.lk/YearWisePdf/2017/252017_E.pdf

61. Ministry of Health, Nutrition, and Indigenous Medicine. National eHealth Guidelines and Standards. 2016 03; Available from: http://www.health.gov.lk/enWeb/publication/NeG S_v_1.pdf

62. Ministry of Health, Nutrition, and Indigenous Medicine. The National Policy on Health Information. 2017; Available from: http://www.health.gov.lk/moh_final/english/publi c/elfinder/files/publications/publishpolicy/Nation alPolicyonHealthInformation.pdf

63. Chapman KRP, Arunatileka SMKD. Teleconsultation roadmap - the path to telemedicine. In: The 12 th IEEE International Conference on e-Health Networking, Applications and Services. The 12th IEEE International Conference on e-Health Networking, Applications and Services; 2010. p. 7480.

64. Kumarawadu SP, Nanayakkara ND, Thavaneswaran B, Tharmakulsingam M, Paramanathan N, Perera L, et al. A Portable Remote Medical Consultation System for the Use of Distant Rural Communities. Rajshahi University Journal of Science and Engineering. 2015;43:81-87. 